\title{
Study of Oil-Water Two-Phase Stratified Flow in Horizontal Fractures
}

\author{
Na Huang, Dongxu Liu, Yuhan Sun, and Lei Liu* \\ State Key Laboratory of Multiphase Flow in Power Engineering, Xi' an Jiaotong University, 710049 Xi' an, China
}

\begin{abstract}
The relative permeability of oil-water two-phase flow is an important parameter in fractured petroleum reservoirs. It is widely accepted that the sum of relative permeabilities is less than 1 . In this study, a series of experiments have been conducted on six rectangular fractures for oil-water two-phase flows. Analytical investigations of the effects of flow rate, aspect ratio, and fracture size on the relative permeability of oil-water two-phase are analysed. Basic fluid flow equations are combined to develop a new analytical model for water-oil two-phase flow in a horizontal fracture. The simulation results predicted by this model are in good agreement with the experimental data. The relative permeability is a function of flow ratio, viscosity ratio, aspect ratio and saturation. It increases as aspect ratio increases if the fracture depths are the same, while it decreases as aspect ratio increases if the fracture widths are identical. Both experiment and model indicate that the sum of relative permeabilities of oil and water is greater than 1 in some cases, different from the accepted view.
\end{abstract}

\section{Introduction}

Porous media are widely used in such fields as resource development engineering, farmland water conservancy engineering, chemical infiltration, biological tissue, etc [1-3]. In order to simplify mathematical calculations and more vividly represent the pore space of porous media, physical models are usually used to replace complex pore space [4]. The capillary bundle model is one of the most common physical models for the porous media. The most classic physical model is the straight capillary bundle model with the same diameter. But the pore space of porous media has different shapes. In order to make porous media better used in various fields, we need to determine the bundle model of other shapes. To establish the model, we should firstly study the single medium, which is the basis of studying porous media. Fractured reservoirs spread all over the world. But fractured reservoir exploitation is difficult [5]. In order to better exploit the reservoir, we need to know the horizontal flow of oil-water two-phase in single fracture. The relative permeability is an important parameter to describe the oil-water two-phase flow in fractured reservoirs [6-7]. Single fracture is the elementary cell of the fractured reservoirs. Therefore, researching the relative permeability of oil-water two-phase in single fracture is essential.

There is still ambiguity about the sum of relative permeabilities in reservoirs. The relative permeability of oil-water two-phase flow in sand column and rock core were studied and show that the sum of the relative permeability of oil and water phases is less than 1 [8-10]. However, Odeh reported that the relative permeability of oil phase can reach 2.4. He attributed this result to a lubrication effect [11]. Ehrlich and AI-Foderie also found that the maximum value of the relative permeability of oil phase exceeds 1 [12-13]. Shad et al. presented the effect of oil viscosity and flow orientation on smooth parallel plates using the steady-state approach introduced by Fourar et al [14]. They observed lubrication effect yielding the relative permeability values higher than 1 if the fracture was oil-wet [15-16]. They only pointed out that the sum of the relative permeability is greater than 1 in the experiment, but no systematic theoretical analysis has been carried out. In this study, we firstly establish the model of the relative permeability of the oil-water two-phase in a single fracture. Then a comparative experiment is designed to study the oil-water two-phase stratified flow in six fractures with rectangular cross-sections. Water and white oil are used as two-phase fluids entering rectangular pexiglas fractures, and the relative permeability is taken as an index to analyse the flow laws in single fractures. At last, theoretical data are compared with experimental data to understand the oilwater two-phase flow in single fractures more deeply.

\section{Theory}

\subsection{Absolute permeability}

According to Darcy-Weisbach equation [17], the pressure drop of single-phase flow in the fracture can be expressed by using equation (1).

\footnotetext{
* Corresponding author: rliu@mail.xjtu.edu.cn
} 


$$
\frac{\Delta p}{L}=\frac{1}{8} \lambda \rho u^{2} \frac{B}{A}
$$

Seepage flow is generally laminar flow, and its Darcy friction coefficient is given by equation (2) [18].

$$
\lambda=\frac{P o}{R e}=\frac{P o \cdot \mu}{u \rho d}
$$

The fracture studied in this paper is fractures with rectangular cross-sections, Po can be calculated as follows [19]:

$$
\text { Po }=96\left(1-1.3553 \frac{H}{W}+1.9467\left(\frac{H}{W}\right)^{2}-1.7012\left(\frac{H}{W}\right)^{3}+0.9546\left(\frac{H}{W}\right)^{4}-0.2537\left(\frac{H}{W}\right)^{5}\right)
$$

For single-phase flow in the fracture, the real average velocity is equal to the seepage velocity. From the above equations, equation (4) is obtained.

$$
J=\frac{32 A^{2}}{P_{o} \cdot B^{2}} \frac{1}{\mu} \frac{\Delta p}{L}
$$

The seepage velocity also can be calculated using the following equation (5) [20].

$$
J=\frac{k}{\mu} \frac{\Delta p}{L}
$$

Therefore, the equation for calculating absolute permeability is obtained.

$$
k=\frac{32}{P_{o}} \frac{A^{2}}{B^{2}}
$$

\subsection{The relative permeability of oil-water two- phase}

Fractures are placed horizontally in the experiment. Assuming a fully developed flow, the integral forms of the momentum equations for the two fluids can be expressed using equation (7), (8) [21].

$$
\begin{aligned}
A S_{o} \frac{\Delta p}{L}-\tau_{o} B_{o} \pm \tau_{i} B_{i} & =0 \\
A S_{w} \frac{\Delta p}{L}-\tau_{w} B_{w} \mathrm{~m} \tau_{i} B_{i} & =0
\end{aligned}
$$

According to equation (7), (8), the following equation can be derived.

$$
\frac{\Delta p}{L}=\frac{\tau_{o} B_{o}+\tau_{w} B_{w}}{A}
$$

The shear stress between the fluid and the fracture surface can be expressed by the following equation [22].

$$
\begin{gathered}
\tau_{o}=\frac{1}{8} \lambda_{o} \rho_{o} u_{o}^{2}=\frac{P o \mu_{o} u_{o} B_{o}}{32 A S_{o}} \\
\tau_{w}=\frac{1}{8} \lambda_{w} \rho_{w} u_{w}^{2}=\frac{P o \mu_{w} u_{w} B_{w}}{32 A S_{w}}
\end{gathered}
$$

For the phase separation model, the relative permeability of oil-water two-phase can be given by equation (12), (13) [23].

$$
\begin{aligned}
& J_{o}=\frac{Q_{o}}{A}=\frac{k_{r o} k}{\mu_{o}} \frac{\Delta p}{L} \\
& J_{w}=\frac{Q_{w}}{A}=\frac{k_{r w} k}{\mu_{w}} \frac{\Delta p}{L}
\end{aligned}
$$

From the equation (9) to equation (13), the relative permeability equation of oil phase and water phase can be expressed as follows.

$$
\begin{gathered}
k_{r o}=\frac{B^{2}}{B_{o}{ }^{2} / S_{o}{ }^{2}+Q_{w} \mu_{w} B_{w}^{2} / Q_{o} \mu_{o} S_{w}^{2}} \\
k_{r w}=\frac{B^{2}}{B_{w}{ }^{2} / S_{w}{ }^{2}+Q_{o} \mu_{o} B_{o}^{2} / Q_{w} \mu_{w} S_{o}^{2}}
\end{gathered}
$$

According to the equation (6), (12), (13), the relative permeability equation of oil phase and water phase also can be expressed as follows.

$$
\begin{gathered}
k_{r o}=\frac{P o Q_{o} \mu_{o} B^{2}}{32 A^{3} \frac{\Delta p}{L}} \\
k_{r w}=\frac{P o Q_{\mathrm{w}} \mu_{w} B^{2}}{32 A^{3} \frac{\Delta p}{L}}
\end{gathered}
$$

The equation (14), (15) show that the relative permeability is a function of flow ratio, viscosity ratio, wet perimeter and saturation. The variation of the relative permeability is revealed by the following experiment.

\section{Experiment}

\subsection{Experimental method}

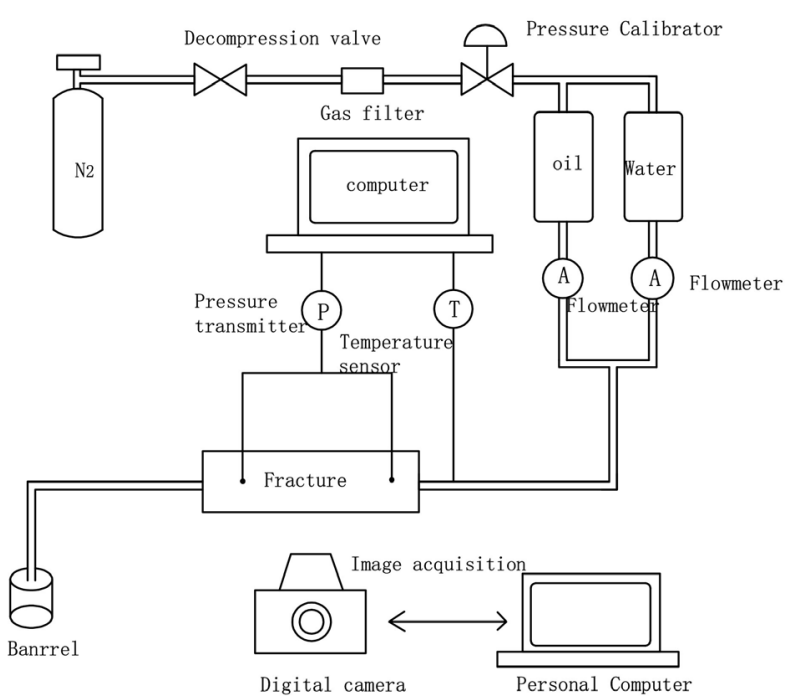

Fig. 1. Flow experiment system

Fig. 1 shows that high-pressure nitrogen is used as provide power for the flow of fluids in fractures. Water 
and white oil are used as two-phase fluids entering rectangular plexiglas fractures. Temperature sensor is used to measure temperatures of flows. Pressure transmitter is used to measure the pressure drop of the fluid flow across fracture. The flow rate of oil phase and water phase are measured by flowmeters. The flow state of oil and water in the fracture is observed by camera. Then the oil saturation was determined by analysing the oil-water depth ratio (Fig. 2), and the average value of experimental value is taken as the oil saturation.

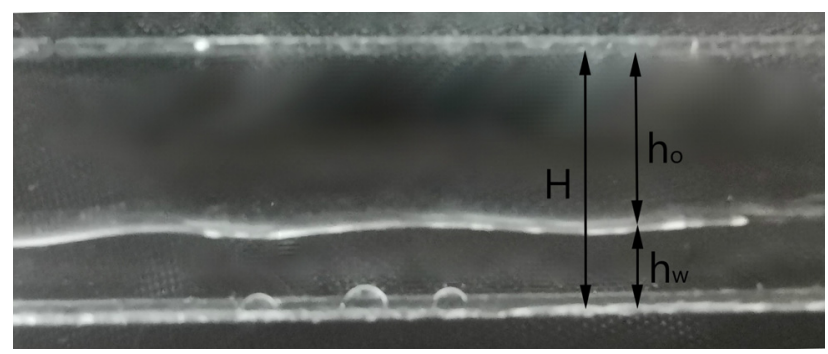

Fig. 2. The stable stratified flow in the fracture

\subsection{Experimental material}

The viscosity and density of white oil measured at $23^{\circ} \mathrm{C}$ are $0.017 \mathrm{~Pa} \cdot \mathrm{s}, 835 \mathrm{~kg} / \mathrm{m}^{3}$, the viscosity and density of water are $0.001 \mathrm{~Pa} \cdot \mathrm{s}, 1000 \mathrm{~kg} / \mathrm{m}^{3}$. The parameters of fractures are shown in Table 1.

Table 1. Fracture parameters

\begin{tabular}{cccc}
\hline $\begin{array}{c}\text { Fracture } \\
\text { number }\end{array}$ & $\begin{array}{c}\text { Width } \\
\text { W/mm }\end{array}$ & $\begin{array}{c}\text { Depth } \\
\text { H/mm }\end{array}$ & $\begin{array}{c}\text { Aspect ratio } \\
\text { H/W }\end{array}$ \\
\hline 1 & 10.0 & 1.15 & 0.115 \\
2 & 1.15 & 10.0 & 8.696 \\
3 & 10.0 & 2.15 & 0.215 \\
4 & 2.15 & 10.0 & 4.651 \\
5 & 10.0 & 4.0 & 0.400 \\
6 & 4.0 & 10.0 & 2.500 \\
\hline
\end{tabular}

\section{Results and discussion}

\subsection{Oil saturation}

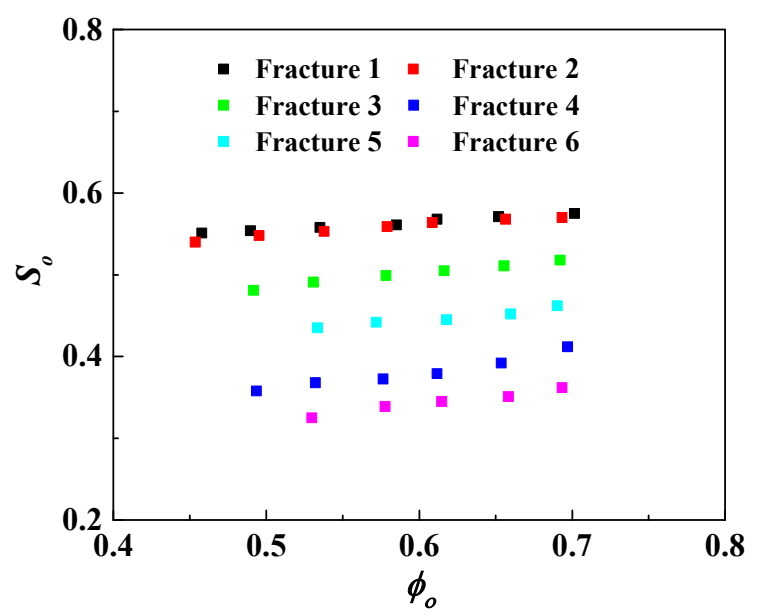

Fig. 3. Relationship between the oil content and oil saturation

Fig. 3 reflects that the oil saturation increases as aspect ratio increases if the fracture depths are identical. While it decreases as aspect ratio increases if the fracture widths are the same. The smaller fracture 1 and 2 have the greater oil saturation. Possible reasons for this result may be demonstrated as follows. On one hand, the sum of oil and water flow is fixed in the experiment, the smaller fractures have larger oil flow velocity, which makes the oil phase not easy to adhere to the fracture surface, and can flow out of the fracture smoothly. On the other hand, the aspect ratio affects the distribution of the oil-water two-phase flow in the fracture[24]. These factors together lead to these phenomena.

\subsection{Pressure drop}

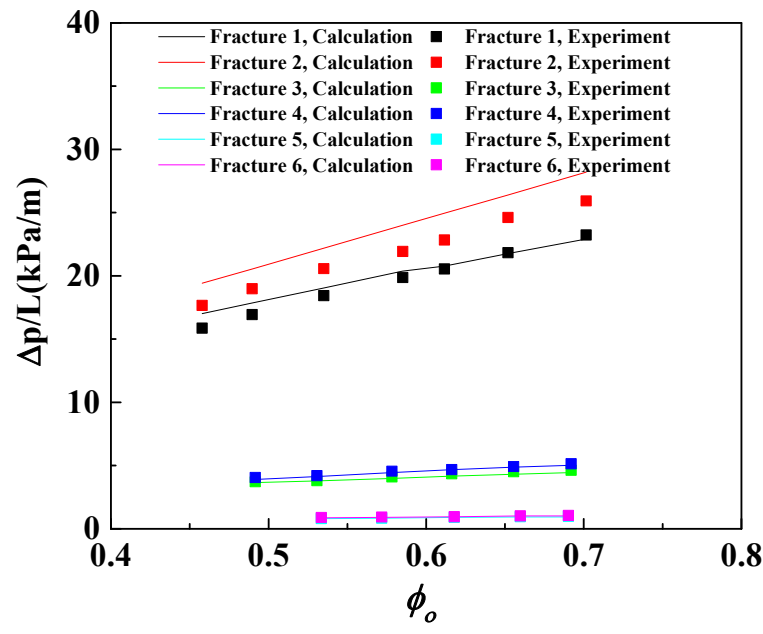

Fig. 4. Pressure drop of oil-water two-phase stratified flow in different fractures

Fig. 4 shows calculation is consistent with our experiment, and the error is less than $15 \%$. The pressure drop of oil-water stratified flow is very small, and the measurement accuracy of pressure sensor is limited, so the error is mainly the instrument measurement error with small pressure drop besides the model itself. Smaller fractures have great influence on the change of pressure drop. The pressure drops of the different fractures are not equal at the same oil content. When the fracture area is the same, fractures with larger aspect ratio have larger pressure drop. The pressure drop increases as aspect ratio increases if the fracture depths are the same, while it decreases as aspect ratio increases if the fracture widths are identical.

$$
\frac{\Delta p}{L}=\frac{P o}{32 A^{3}}\left[\mu_{o} Q_{o}\left(2 H+\frac{W}{S_{o}}\right)^{2}+\mu_{w} Q_{w}\left(2 H+\frac{W}{S_{w}}\right)^{2}\right]
$$

From equation (9), the equation (18) is obtained. It is can be seen from the equation (18) that the smaller fracture is, the larger Po number[19], which may lead to the greater pressure drop. So the smaller fractures have great influence on the change of pressure drop, which is consistent with our experimental phenomenon. When the fracture area is the same, oil saturation of fracture with smaller aspect ratio is greater than that of fracture with larger aspect ratio. The depth of the fracture with larger 
aspect ratio is much higher than that of the fracture with smaller aspect ratio, so fractures with larger aspect ratio have larger pressure drop. For a certain fracture depth, the depth is much greater than the width, area and Po number becomes the main factors. The area decreases, Po number increases as the aspect ratio increases. So the pressure drop increases as aspect ratio increases if the fracture depths are the same. For a certain fracture width, the width is much greater than the depth, the area increases, and Po number decreases as the aspect ratio increases. It makes pressure drop decrease with the increase aspect ratio.

\subsection{Relative permeability}

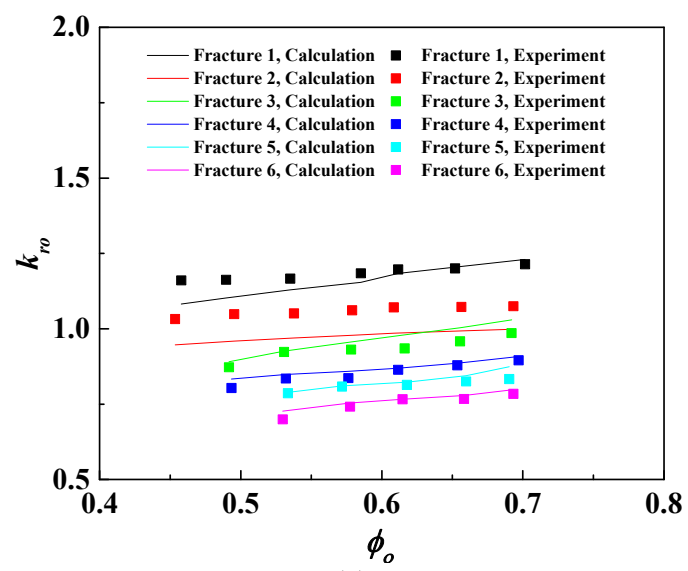

(a)

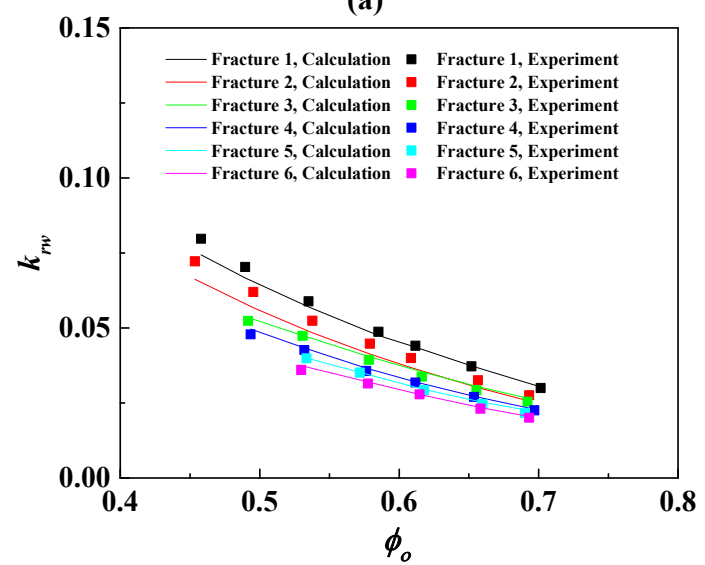

(b)

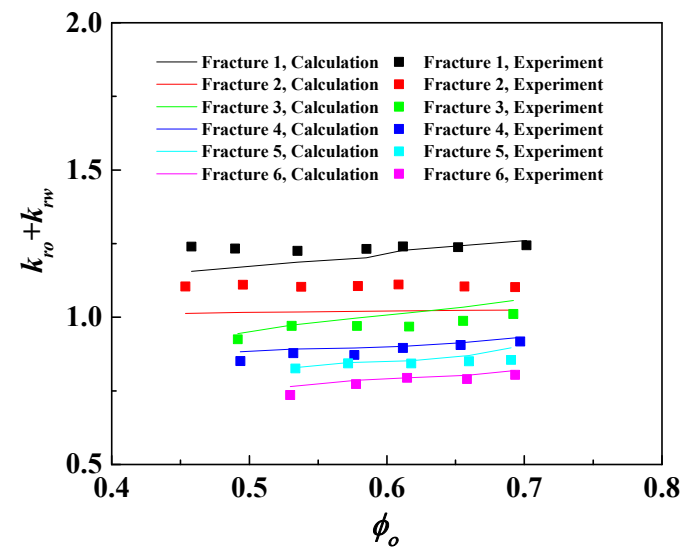

(c)

Fig. 5. The relative permeability of oil-water two-phase stratified flow in different fractures
As can be seen from Fig. 5, the calculation is in good agreement with the experiment, and the error is less than $15 \%$. The relative permeability of water phase gradually decreases, and its floating value is small. The relative permeability of oil phase increases slowly. The flow of water phase in the fracture is continuous in oil-water two-phase flow, and the dynamic viscosity of water is small. So the relative permeability of water phase changes little in the whole oil content range. When the oil-water two-phase flow forms stable stratified flow, the increase of oil content leads to the gradual increase of oil flow rate, which makes the oil phase flow more easily in the fracture, and the oil-water two-phase flow is continuous in the fracture when the stratified flow is stable, so the relative permeability of oil phase increases slowly.

The relative permeability of oil-water two-phase increases as aspect ratio increases if the fracture depths are the same, while it decreases as aspect ratio decreases if the fracture widths are identical. For fractures with equal cross-sectional areas, fractures with smaller aspect ratio have the larger relative permeability. Equations (16) and (17) show pressure drop is inversely proportional to the relative permeability. According to the analysis of pressure difference in section 4.2, the pressure drop increases as aspect ratio increases if the fracture depths are the same, while it decreases as aspect ratio increases if the fracture widths are identical. For fractures with equal cross-sectional areas, fractures with the larger aspect ratio have the larger pressure drop. So these factors lead to these phenomena. The experiment indicates the relative permeability is related to aspect ratio. The equation (14), (15) show that the relative permeability is a function of flow ratio, viscosity ratio, wet perimeter and saturation. Wet perimeter is a function of saturation, depth and width, and the relative permeability is also a function of flow ratio, viscosity ratio, aspect ratio and saturation. So both experiment and theory show that the relative permeability is related to aspect ratio.

The smaller fractures have the larger relative permeability. Fracture surface is hydrophobic and lipophilic, water flows in fracture to form a layer of water film on the wall of fracture, and oil is easy to adhere to the fracture. The sum of oil-water two-phase flow is constant in the experiment, the smaller fractures have larger flow velocity which enable oil and water to pass through easily. According to the equation (14), (15), Po number and area is the main factors which influence the relative permeability. The smaller fractures have the larger Po number and smaller area, so the smaller fractures have the larger relative permeability. It also indicates that the relative permeability is related to the speed of oil and water injection.

It also shows that the relative permeability of oil phases in fractures is greater than 1 in smaller fractures. On the one hand, the sum of oil and water flow is fixed in the experiment, the smaller fracture has a larger oil flow velocity, which makes the oil flow out of the fracture smoothly. On the other hand, water has lubrication effect on oil phase[11], and the viscosities of oil phase and water phase are different and have the 
larger viscosity ratio. Water phase will promote the flow of oil phase when oil and water flow together. These factors make the relative permeability of oil phase greater than 1. From equation (14), (15), we obtain that the relative permeability is a function of flow ratio, viscosity ratio, wet perimeter and saturation. If the oilwater flow ratio and the oil-water viscosity ratio is large, the relative permeability of the oil phase will be greater than 1. So experimental results and theoretical calculation both show that the sum of relative permeabilities is greater than 1 in some cases.

\section{Conclusion}

Different fractures have different oil saturation. Flow velocity makes the difficulty of oil-water two-phase flow through fractures different. The aspect ratio will affect the wetted perimeter of oil phase and water phase, so these factors jointly affect the oil saturation.

The model shows the relative permeability is a function of flow ratio, viscosity ratio, wet perimeter and saturation. Wet perimeter is a function of saturation, depth and width, so the relative permeability is also a function of flow ratio, viscosity ratio, aspect ratio and saturation.

The relative permeability of oil-water two-phase increases as aspect ratio increases if the fracture depths are the same, while it decreases as aspect ratio increases if the fracture widths are identical. For fractures with equal cross-sectional areas, fractures with different aspect ratios have different relative permeability. Both theory and experiment indicate that the relative permeability is related to aspect ratio.

The smaller fractures have the larger relative permeability. These fractures have the larger flow velocity, which enables oil and water to pass through easily. It also indicates that the relative permeability is related to the speed of oil and water injection.

The simulation results predicted by this model are in good agreement with the experimental data. Experiment and calculation show the sum of the relative permeability is greater than 1 in some cases.

\section{Acknowledgment}

The authors are grateful for financial support from the National Natural Science Foundation of China (Grant No. 11572241).

\section{Nomenclature}

$J$ Seepage velocity, $\mathrm{m} / \mathrm{s}$

$J_{o}$ Seepage velocity of oil, $\mathrm{m} / \mathrm{s}$

$J_{w}$ Seepage velocity of water, $\mathrm{m} / \mathrm{s}$

$u$ Mean velocity, $\mathrm{m} / \mathrm{s}$

$u_{o}$ Mean velocity of oil, $\mathrm{m} / \mathrm{s}$

$u_{w}$ Mean velocity of water, $\mathrm{m} / \mathrm{s}$

$\mu$ Dynamic viscosity, Pa.s

$\mu_{o}$ Dynamic viscosity of oil, Pa.s

$\mu_{w}$ Dynamic viscosity of water, Pa.s $\rho$ Density, $\mathrm{kg} / \mathrm{m}^{3}$

$\rho_{o}$ Density of oil, $\mathrm{kg} / \mathrm{m}^{3}$

$\rho_{w}$ Density of water, $\mathrm{kg} / \mathrm{m}^{3}$

$\lambda$ Friction coefficient

$\lambda_{o}$ Friction coefficient of oil phase

$\lambda_{w}$ Friction coefficient of water phase

$B$ Wetted perimeter, $\mathrm{m}$

$B_{o}$ Wetted perimeter of oil phase, $\mathrm{m}$

$B_{w}$ Wetted perimeter of water phase, $\mathrm{m}$

$B_{i}$ Wetted perimeter of oil-water interface, $\mathrm{m}$

$k$ Absolute permeability

$k_{r o}$ The relative permeability of oil phase

$k_{r w}$ The relative permeability of water phase

$S_{o}$ Oil saturation

$S_{w}$ Water saturation

$Q_{o}$ Volume flow of oil phase, $\mathrm{m}^{3} / \mathrm{s}$

$Q_{w}$ Volume flow of water phase, $\mathrm{m}^{3} / \mathrm{s}$

$\tau_{0}$ Shear stress between oil phase and surface, $\mathrm{Pa}$

$\tau_{w}$ Shear stress between water phase and surface, $\mathrm{Pa}$

$\tau_{i}$ Shear stress of oil-water interface, $\mathrm{Pa}$

$H$ Depth of rectangular fracture, $\mathrm{m}$

Po Poiseuille number

Re Reynolds number

$W$ Width of rectangular fracture, $\mathrm{m}$

$\phi_{o}$ Oil content

$\triangle p$ Pressure drop, $\mathrm{Pa}$

$L$ Fracture length, $\mathrm{Pa}$

\section{References}

1 Anonymous, AIP Conf. Proc. 1254 (2010).

2 D. Magliacano, M. Ouisse, A. Khelif, S. De Rosa, F. Franco, N. Atalla, M. Collet, Mech. Syst. Signal Pr. 142, 16 (2020).

3 F.Y. Wang, H. Cheng, J. Petrol. Sci. Eng. 188, 9 (2020).

4 M.Z. Dong, F.A.L. Dullien, L.M. Dai, D.M. Li, Transport Porous Med. 59 (1), 1 (2005).

5 X. Zhou, Y.C. Wang, L.H. Zhang, K.W. Zhang, Q. Jiang, H.B. Pu, L. Wang, Q.W. Yuan, Fuel 272, 12 (2020).

6 F. Torabi, N. Mosavat, O. Zarivnyy, Fuel 163, 196 (2016).

7 N. Zhang, B.C. Yan, Q. Sun, Y.H. Wang, J. Petrol. Sci. Eng. 154, 382 (2017).

8 M.J. Blunt, M.D. Jackson, M. Piri, P.H. Valvatne, Adv. Water Resour. 25 (8-12), 1069 (2002).

9 W. Hu, S.L. Yang, G.F. Liu, Z.L. Wang, P. Wang, H. Lei, Transport Porous Med. 109 (3), 527 (2015).

10 J. Hou, F.Q. Luo, D.G. Wang, Z.Q. Li, S.X. Bing, Energ. Fuel 26 (7), 4300 (2012).

11 A.S. Odeh, Trans. Am. Inst. Min. Metall. Eng. 216, 346 (1959).

12 R. Ehrlich, Transport Porous Med. 11 (3), 201 (1993). 
13 D. Al-Foderie, R. Al-Sayegh, M. Omar, 2015 International Conference on Sustainable Mobility Applications, Renewables and Technology (SMART), 7 pp. (2015).

14 M. Fourar, S. Bories, R. Lenormand, P. Persoff, Water Resour. Res. 29 (11), 3699 (1993).

15 S. Shad, I.D. Gates, J. Can. Petrol. Technol. 49 (2), 48 (2010).

16 S. Shad, B.B. Maini, I.D. Gates, Int. J. Multiphas. Flow 57, 78 (2013).

17 E.G. Donskov, V.P. Lyalyuk, A.D. Donskov, Steel Trans. 43 (4), 197 (2013).

18 R.L. Daugherty, A.C. Ingersoll, Fluid mechanics. (McGraw-Hill, 1954).

19 R.K. Shah, A.L. London, F.M. White, J. Fluid Eng. 102 (2), 431 (1980).

20 R.A. Wooding, H.J. Morelseytoux, Annu. Rev. Fluid Mech. 8, 233 (1976).

21 G.B. Wallis, One-dimensional two-phase flow. (McGraw-Hill, 1969).

22 N. Brauner, D.M. Maron, J. Rovinsky, Int. J. Multiphas. Flow 24 (6), 975 (1998).

23 Higdon, J.L. J, J. Fluid Mech. 730, 1 (2013).

24 J.Y. Xu, Y.X. Wu, Y. Chang, J. Guo, Chem. Eng. Technol. 31 (10), 1536 (2008). 